\title{
Pie diabético en los tiempos del Covid-19
}

\section{Diabetic foot in the times of Covid-19}

\section{Sr. Editor:}

La pandemia del COVID-19 viene desafiando drásticamente la integridad de los sistemas de salud en más de 100 países alrededor del mundo incluyendo al Perú (1); a pesar de ello, se suman las enfermedades crónicas que generan una permanente carga de enfermedad en nuestro país y que requieren una constante atención médica como la Diabetes Mellitus (11\%; aprox. 3520000 personas) y sus complicaciones tardías como el pie diabético (2).

Los gobiernos vienen implementando una serie de medidas de salud pública sin precedentes con el objeto de reducir la curva de infección y tasas de letalidad por el COVID-19; para ello se está priorizando la atención médica de los infectados, suspendiendo o cancelando los servicios médicos "no esenciales", incluyendo a los procedimientos electivos $(3,4)$. Esto en el caso de los pacientes con pie diabético ha interrumpido el flujo de atención, conllevando a que los procedimientos relacionados al salvataje del pie diabético no se realicen, por ser erróneamente clasificados como no esenciales (5).

Ante esta inusual problemática, diversas organizaciones nacionales e internacionales que velan por el salvataje del pie diabético, han publicado una

Tabla 1. Modelo de triaje propuesto para pacientes con pie diabético en la pandemia del COVID-19.

\begin{tabular}{|c|c|c|c|}
\hline TRIAJE & CONDICIONES & $\begin{array}{l}\text { LUGAR DE } \\
\text { ATENCIÓN }\end{array}$ & URGENCIA \\
\hline $\begin{array}{l}\text { CRÍTICO } \\
\text { (0,25\% de pacientes } \\
\text { diabéticos) }\end{array}$ & $\begin{array}{l}\text { - IDSA moderado-severo } \\
\text { - Necrosis gaseosa } \\
\text { - SIRS/Sepsis } \\
\text { - Isquemia crónica que amenaza la extremidad (CLTI) }\end{array}$ & Hospital & $\begin{array}{l}\text { Prioridad } 1 \\
\text { URGENCIA }\end{array}$ \\
\hline $\begin{array}{l}\text { GRAVE } \\
(0,75 \% \text { de los } \\
\text { pacientes diabéticos })\end{array}$ & $\begin{array}{l}\text { - IDSA leve-moderado } \\
\text { - Isquemia crónica que amenaza la extremidad (CLTI) } \\
\text { - Necrosis seca } \\
\text { - Empeoramiento de la úlcera del pie } \\
\text { - Pie de Charcot activo }\end{array}$ & $\begin{array}{l}\text { Consultorio } \\
\text { ambulatorio con } \\
\text { laboratorio. } \\
\text { Centro de cirugía } \\
\text { Consultorio de } \\
\text { podología }\end{array}$ & Prioridad 2 \\
\hline $\begin{array}{l}\text { DOMICILIARIO } \\
\text { (3\% de los pacientes } \\
\text { diabéticos) }\end{array}$ & $\begin{array}{l}\text { - Ulcera en mejoría } \\
\text { - Pie de Charcot no activo (sin calzado estable) }\end{array}$ & $\begin{array}{l}\text { Consultorio de } \\
\text { podología } \\
\text { Telemedicina } \\
\text { En casa }\end{array}$ & Prioridad 3 \\
\hline $\begin{array}{l}\text { ESTABLE } \\
\text { (94\% de los } \\
\text { pacientes } \\
\text { diabéticos) }\end{array}$ & $\begin{array}{l}\text { - Úlcera venosa no complicada de la pierna } \\
\text { - Úlcera de pie recientemente curada } \\
\text { - Pie de Charcot inactivo (con calzado estable) } \\
\text { - Amputación curada } \\
\text { - Evaluaciones de riesgo del pie diabético }\end{array}$ & $\begin{array}{l}\text { Telemedicina } \\
\text { En casa }\end{array}$ & Prioridad 4 \\
\hline
\end{tabular}

Fuente: Quispe Y, Manrique H, Sáenz S, et al. (6).

Departamento de Cirugía de Tórax y Cardiovascular, Hospital Nacional Edgardo Rebagliati Martins. Lima, Perú.

Médico Residente, Maestro en Gerencia de Servicios de Salud.

Médico Cirujano Cardiovascular y Endovascular. 
serie de recomendaciones que replantean el sistema de triaje hacia una atención pandémica estándar para el pie diabético en riesgo $(5,6)$. Entre ellas, destaca la recientemente publicada por Rogers et al. (5), y traducida al español por Quispe Y. et al. (6) (tabla 1), donde se estratifica la evaluación del pie diabético basándose en el score de severidad planteado por el Infectious Diseases Society of America (IDSA); y además, resalta el papel que desempeña la podiatría y esta condición en el contexto de la pandemia del COVID-19.

Pacientes con pie diabético complicado con necrosis gaseosa, signos evidentes de sepsis (IDSA moderadosevero), isquemia aguda o crónica que amenaza la extremidad CLTI (por sus siglas en inglés Chronic Limb-Threatening Ischemia) son tributarios de manejo clínico-quirúrgico urgente y estrictamente hospitalario (prioridad 1). Por otro lado, sepsis asociada a un IDSA leve-moderado, necrosis seca, lesión ulcerosa complicada, CLTI y pie de Charcot activo (inflamación e inestabilidad) deben obtener una atención por consultorio o procedimiento quirúrgico ambulatorio (prioridad 2). Asimismo, lesiones ulcerosas en proceso de mejoría y un pie de Charcot inactivo, se podrían optar por una evaluación médica por consultorio, vía telemedicina o en casa (prioridad 3); y finalmente, lesiones ulcerosas recientemente curadas o de origen venoso, herida curada por amputación, pie de Charcot inactivo y las evaluaciones de riesgo para pie diabético, son atenciones meramente por vía telemedicina o en casa (prioridad 4) $(5,6)$.

Es evidente la necesidad de implementar y adoptar un sistema de triaje para el pie diabético en la actual pandemia del COVID-19, el cual permita mediante una atención hospitalaria oportuna, consultas ambulatorias, visitas domiciliarias y la telemedicina desde casa, reducir la carga de pacientes sobre el sistema de atención médica, evitar la exposición innecesaria al COVID-19 y aminorar el riesgo de pérdida de la extremidad.

\section{W Samir Cubas ${ }^{1, a}$, Manolo Briceño-Alvarado ${ }^{1, b}$, Félix Tipacti-Rodríguez ${ }^{1, b}$}

\section{Correspondencia:}

\section{W Samir Cubas}

Departamento de Cirugía de Tórax y Cardiovascular, Hospital Nacional Edgardo Rebagliati Martins.

Av Edgardo Rebagliati 490, Jesús María, Lima, Perú. Correo electrónico: wsamircubas@gmail.com

\section{REFERENCIAS BIBLIOGRÁFICAS}

1. Google. Mapa del coronavirus (COVID-19). California: Google; 2010. (Citado el 04 de agosto del 2020. Disponible en: https://google.com/covid19map/?hl=es-419

2. Carrillo-Larco RM, Bernabé-Ortiz A. Diabetes mellitus tipo 2 en Perú: una revisión sistemática sobre la prevalencia e incidencia en población general. Rev Peru Med Exp Salud Publica. 2019; 36:26-36.

3. Adalja AA, Toner E, Inglesby TV. Priorities for the US Health Community Responding to COVID-19. JAMA. 2020; 323(14):1343-4.

4. Iacobucci G. Covid-19: all non-urgent elective surgery is suspended for at least three months in England. BMJ. 2020; 368:m1106.

5. Rogers L, Lavery L, Joseph W, Armstrong D. All Feet On Deck-The Role of Podiatry During the COVID-19 Pandemic: Preventing hospitalizations in an overburdened healthcare system, reducing amputation and death in people with diabetes. J Am Podiatr Med Assoc.2020.

6. Quispe Y, Manrique H, Sáenz S, et al. Manual de recomendaciones de la alianza para el salvataje del pie diabético Perú para el manejo de pacientes con diabetes y pie diabético frente al COVID19 PERÚ2020. 1ra ed. Lima, Perú: ALPIEPERÚ; 2020. 30 p.
Recibido: 23/04/2020

Aceptado: 18/08/2020 